\title{
Fusion Suppression and Sub-Barrier Breakup of Weakly Bound Nuclei
}

\author{
D. J. Hinde, ${ }^{1}$ M. Dasgupta, ${ }^{1}$ B. R. Fulton, ${ }^{2}$ C. R. Morton, ${ }^{1}$ R. J. Wooliscroft,${ }^{3}$ A. C. Berriman, ${ }^{1}$ and K. Hagino ${ }^{4}$ \\ ${ }^{1}$ Department of Nuclear Physics, Research School of Physical Sciences and Engineering, Australian National University, \\ Canberra, ACT 0200, Australia \\ ${ }^{2}$ Department of Physics, University of York, York Y010 5DD, United Kingdom \\ ${ }^{3}$ School of Physics and Astronomy, University of Birmingham, Birmingham B15 2TT, United Kingdom \\ ${ }^{4} Y u k a w a$ Institute for Theoretical Physics, Kyoto University, Kyoto 606-8502, Japan
}

(Received 1 May 2002; published 17 December 2002)

\begin{abstract}
The mechanism for the large suppression of complete fusion in the ${ }^{9} \mathrm{Be}+{ }^{208} \mathrm{~Pb}$ reaction has been investigated through measurement of sub-barrier breakup of ${ }^{9} \mathrm{Be}$. Excluding breakup through the ${ }^{8} \mathrm{Be}$ ground state, whose lifetime is too long, a prompt breakup component remains, having sufficient probability to explain the observed suppression of complete fusion. This appears to be associated with interactions at the nuclear surface. The fusion suppression is predicted to be almost proportional to the charge of the target nucleus, making it most significant in reactions with heavy nuclei.
\end{abstract}

DOI: 10.1103/PhysRevLett.89.272701

PACS numbers: 25.70.Jj, 25.70.Mn

Fusion with radioactive nuclei allows the formation and study of the properties of nuclei inaccessible through the use of stable isotopes. For fusion to occur, the fusion barrier resulting from the sum of the repulsive Coulomb and attractive nuclear potentials must be overcome. Measurements of fusion cross sections can thus give information on the nuclear potential, expected to extend to large radii for very weakly bound nuclei. However, the weak binding also results in breakup, and the effects of this on fusion must also be accounted for [1,2].

Studies with weakly bound stable nuclei $[3,4]$ currently allow more precise and extensive experimental measurements. For the reaction of the nucleus ${ }^{9} \mathrm{Be}$ with ${ }^{208} \mathrm{~Pb}$, precision data showed [3] that complete fusion, defined as absorption of all the charge of the projectile, is significantly suppressed. This was attributed to the low binding energy of ${ }^{9} \mathrm{Be}$, which breaks up into charged fragments: ${ }^{9} \mathrm{Be} \rightarrow \alpha+\alpha+n, \quad Q=-1.57 \mathrm{MeV} ;{ }^{9} \mathrm{Be} \rightarrow \alpha+{ }^{5} \mathrm{He}$, $Q=-2.47 \mathrm{MeV}$. Cross sections at above-barrier energies were only $0.68 \pm 0.07$ of those calculated with a barrier-passing model, constrained to reproduce the measured [3] fusion barrier energy of $E_{\text {c.m. }}=38.3 \pm$ $0.3 \mathrm{MeV}$. The missing yield was found [3] in incomplete fusion products, where only one $\alpha$ particle had fused.

A recent model [5] showed qualitatively that coupling to channels leading to breakup enhances fusion below the fusion barrier, but suppresses it above. However, realistic modeling of the effect of breakup on fusion is extremely complex, requiring incorporation of excitation mechanisms, breakup lifetimes, subsequent motion of the fragments, and probabilities of absorption of one or both fragments. Such a model will be needed to interpret future measurements of fusion with weakly bound radioactive nuclei. Isolation of the breakup itself from the probability of fragment capture simplifies the problem. This can be achieved experimentally by measuring breakup at sub-barrier energies, where fragment absorption is suppressed.
In this Letter it is shown that (i) prompt breakup of ${ }^{9} \mathrm{Be}$, which can affect the fusion process, is easily separated experimentally from the long-lived ${ }^{8} \mathrm{Be}$ ground-state breakup; (ii) the dependence of the breakup probability on the separation of the two nuclei can be directly obtained from the breakup differential cross sections; (iii) the sub-barrier prompt breakup probabilities, when extrapolated to the smaller separation distances at abovebarrier energies, appear to be large enough to explain the measured fusion suppression.

Beams of ${ }^{9} \mathrm{Be}$ with energies from 30 to $40 \mathrm{MeV}$, from the 14UD electrostatic accelerator at the Australian National University, bombarded a target of ${ }^{208} \mathrm{~Pb}$, $420 \mu \mathrm{g} \mathrm{cm}^{-2}$ in thickness evaporated onto a $\sim 15 \mu \mathrm{g} \mathrm{cm}^{-2}{ }^{\text {nat }} \mathrm{C}$ backing, and also a $1.0 \mathrm{mg} \mathrm{cm}^{-2}$ selfsupporting ${ }^{n a t} \mathrm{~Pb}$ target. Because of the $\mathrm{C}$ backing, and a proportionately thicker oxide layer, the ${ }^{208} \mathrm{~Pb}$ target gave more $\alpha$ particles from low- $Z$ contaminants, and thus the data presented come largely from the ${ }^{n a t} \mathrm{~Pb}$ target. Measurements were also made with $\mathrm{C}$ and ${ }^{7} \mathrm{LiO}_{2}$ targets to allow estimation of the contaminant $\alpha$-particle spectra. Three rectangular detector telescopes comprising $69 \mu \mathrm{m}$ and $500 \mu \mathrm{m}$ thick Si surface barrier detectors [6], gave particle energies and identification. Each detector subtended $\pm 6.3^{\circ}$ (in-plane) by $\pm 1.4^{\circ}$ with respect to the center of the detector. Measurements were made from $70^{\circ}$ to $172^{\circ}$ at 30,34 , and $36 \mathrm{MeV}$, and from $120^{\circ}$ to $172^{\circ}$ at 32,38 , and $40 \mathrm{MeV}$. Normalization of the cross sections was achieved using monitor detectors at $\pm 15^{\circ}$, together with Rutherford scattering measurements at lower energies.

Breakup of the projectile-like nucleus results in two $\alpha$ particles, which are kinematically focused inside a cone, due to their common source [7]. The limited detector angular acceptance meant that usually only one of these was detected, thus contributing to the $\alpha$-singles spectrum. When both alphas entered a detector (doubles), the event appeared in a distinct group in the $\Delta E-E$ 
matrix, having a $\Delta E$ signal and total energy associated with both $\alpha$ particles. Count rates were low, resulting in no contamination from random doubles events.

The $\alpha$-singles energy spectra for selected beam energies, observed at $166^{\circ}$ from the ${ }^{\text {nat }} \mathrm{Pb}$ target, are shown in Figs. 1(a)-1(c), as raw data (upper spectra) and with low energy contaminant subtraction (thin line histograms). Discrete peaks between 7 and $9 \mathrm{MeV}$ in Fig. 1(a) result from $\alpha$ decay of complete and incomplete fusion products. All spectra show a 3-4 MeV wide peak at an energy corresponding to the projectile velocity, superimposed on a broader background, which remains after contaminant subtraction. The subtraction hardly affects the spectra at and above the energy of the beam-velocity peaks.

To compare the total breakup cross sections with those for fusion, the angular distributions of the singles (after contaminant subtraction) and doubles $\alpha$ particles were extrapolated to more forward angles, guided by distortedwave Born approximation (DWBA) calculations [8]. The yield forward of $70^{\circ}$ contributed $\sim 20 \%$ to the total cross section. The shapes of the angular distributions change slowly with beam energy, giving confidence that cross sections could be estimated where the data extended only to $120^{\circ}$. A $20 \%$ rather than $10 \%$ extrapolation uncertainty was assigned to these points. Since breakup of ${ }^{9} \mathrm{Be}$ results in two $\alpha$ particles, the observed breakup cross sections were obtained by summing the doubles and half the $\alpha$-singles yields. At the highest energies, where fusion starts to become significant (see Fig. 2), capture of one or both of these $\alpha$ particles causes underestimation of the

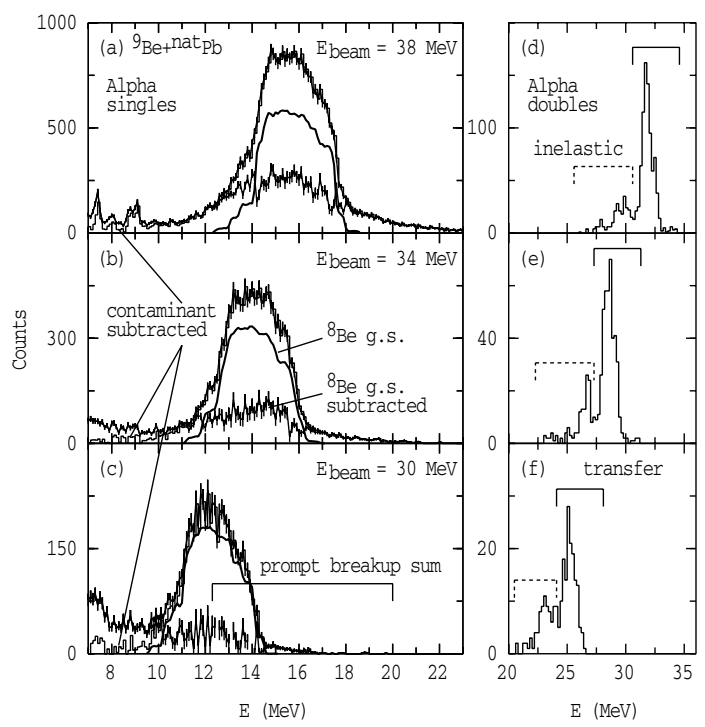

FIG. 1. Energy spectra of singles $\alpha$ particles (left panels) and corresponding $\alpha$ doubles (right), observed at $166^{\circ}$, for the indicated beam energies incident on the ${ }^{n a t} \mathrm{~Pb}$ target. The predicted singles spectra resulting from the observed doubles events, assuming ${ }^{8} \mathrm{Be}$ g.s. decay, are shown by the thick line histograms in the left panels, and the spectrum after subtraction of this component is labeled ${ }^{8} \mathrm{Be}$ g.s. subtracted. actual breakup occurring. The extracted breakup cross sections are shown in Fig. 2, together with the measured incomplete fusion and the measured and calculated complete fusion cross sections [3]. The deficit of the measured complete fusion over that calculated is clearly seen. Although the sub-barrier breakup cross sections are very large, not all the breakup observed can influence the reaction processes at the fusion barrier. This is because the breakup consists of two components: (i) prompt projectile breakup, and (ii) long-lived ${ }^{8} \mathrm{Be}$ g.s. decay, following transfer or excitation of ${ }^{9} \mathrm{Be}$. The ${ }^{8} \mathrm{Be}$ g.s. lifetime of $\simeq 10^{-16} \mathrm{~s}$ allows the interaction with the target nucleus to take place long before the ${ }^{8} \mathrm{Be}$ breaks up. Thus, only prompt breakup can play a role in the suppression of complete fusion, making it essential to separate it from the long-lived component.

Information on the ${ }^{8} \mathrm{Be}$ g.s. component can be obtained from the $\alpha$-doubles events. The energy spectra of detected ${ }^{8} \mathrm{Be}$ events from ${ }^{n a t} \mathrm{~Pb}$ at $166^{\circ}$ are shown in Figs. 1(d)1(f). The spectra from ${ }^{208} \mathrm{~Pb}$ showed similar features and yields, though with poorer statistics. Each spectrum shows two features, a group of peaks at the high kinetic energies (brackets) separated from a lower energy component (dashed brackets) by a marked dip. The high energy peaks are attributed to neutron stripping [7] to states in $\mathrm{Pb}$, followed by decay of ${ }^{8} \mathrm{Be}$. The bulk of this yield has $Q$ values between +2.0 and $-0.5 \mathrm{MeV}$. A contribution from transfer in the lower energy component cannot be excluded, but it will be shown to be consistent with projectile excitation, followed by breakup. The most important questions are the following: (i) Are these doubles events due to decay of the ${ }^{8} \mathrm{Be}$ ground state (thus being incapable of suppressing fusion)? (ii) More importantly, do they exhaust the singles $\alpha$-particle yields?

To answer these questions, a Monte Carlo simulation was made, not only of the doubles detection efficiency but

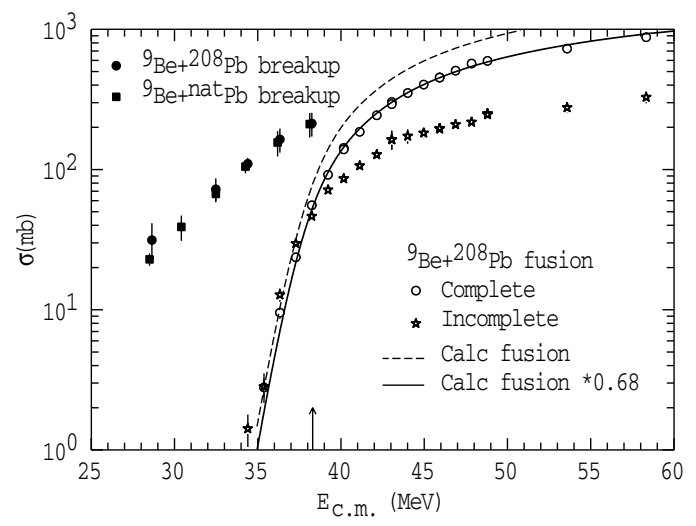

FIG. 2. The estimated total breakup cross sections from this work are shown by the filled points. The measured cross sections for complete and incomplete fusion, and the calculated fusion without breakup, are from Ref. [3]. The arrow marks the measured average fusion barrier energy. 
also of the energy spectrum of the singles $\alpha$ particles. For ${ }^{8} \mathrm{Be}$ ground-state decay, the $Q$ value is only $0.092 \mathrm{MeV}$, and thus the two $\alpha$ particles lie inside a narrow cone (half-angle $\simeq 6^{\circ}$ ). The probability of detecting both these alphas is calculated to be $\sim 20$ times greater than those from prompt breakup, which have a larger relative kinetic energy, and thus wider cone angle. The $\alpha$-singles energy spectra for the two processes are also very different. For ${ }^{8}$ Be with kinetic energy $E$ in the laboratory frame, isotropic breakup with $\alpha$-particle total kinetic energy $T$ in the ${ }^{8} \mathrm{Be}$ rest frame results in a flat-topped singles $\alpha-$ energy spectrum, centered at $(E+T) / 2$, with maximum width $2 \sqrt{E T}$. Decay from the ${ }^{8} \mathrm{Be}$ ground state $(T=$ $0.092 \mathrm{MeV}$ ) for $E=28 \mathrm{MeV}$ gives a peak width of 3.2 $\mathrm{MeV}$ - similar to the peak widths observed. For prompt breakup, taking $T=1 \mathrm{MeV}$, the width is $11 \mathrm{MeV}$. Thus, a narrow peak in the $\alpha$-singles energy spectrum corresponding to the beam velocity is characteristic of ${ }^{8} \mathrm{Be}$ g.s. decay.

Assuming that the doubles events resulted exclusively from ${ }^{8} \mathrm{Be}$ g.s. decay, the distribution of energies $E$ of the $\alpha$-doubles events [Figs. 1(d) -1 (f)] were fed into the Monte Carlo code to predict the corresponding $\alpha$-singles energy spectra for each beam energy and detector angle. These are represented in Figs. 1(a)-1(c) by the thick line histograms, which reproduce the beam-velocity peaks. Thus the observed $\alpha$-doubles events are indeed from ${ }^{8} \mathrm{Be}$ g.s. decay, as is the beam-velocity peak in each singles energy spectrum. This large component, resulting from transfer and inelastic reactions, has significant implications for systematic studies [9] of breakup cross sections. After subtracting the ${ }^{8} \mathrm{Be}$ g.s. component, the $\alpha$-singles energy spectrum remaining is much broader than the ${ }^{8} \mathrm{Be}$ g.s. component, and hence must be associated with decays having a higher $\alpha$ kinetic energy and $Q$ value. A much shorter lifetime can be expected, and this prompt breakup component may well be responsible for the suppression of complete fusion at above-barrier energies.

To investigate this, the breakup probabilities for each mechanism were determined from the ratios of the measured differential cross sections to those for Rutherford scattering at each energy and angle. Those for ${ }^{8} \mathrm{Be}$ g.s. decay resulting from neutron stripping reactions and projectile excitation were determined from the high and low energy components, respectively, in the $\alpha$-doubles spectra (see Fig. 1). These were corrected for the calculated doubles detection efficiency. The differential cross sections for prompt breakup, identified with the broad peak in the subtracted singles $\alpha$-particle energy spectrum, were obtained by summing the counts from the peak position upwards [see Fig. 1(c)]. This avoids uncertainties due to subtraction of the low energy contaminant alphas, and taking only half the spectrum accounts for the production of two $\alpha$ particles in breakup. The breakup probabilities are shown in Fig. 3 as a function of the distance of closest approach $\left(R_{\min }\right)$ of the nuclei on
Coulomb trajectories. Error bars indicated are statistical only, and an additional $10 \%$ uncertainty is estimated for the prompt breakup, because of the subtraction of the ${ }^{8} \mathrm{Be}$ g.s. decay components. The full lines are least-squares exponential fits to the data, omitting those points at the smallest $R_{\min }$ values, where fusion depletes the yields.

The probabilities for neutron transfer from ${ }^{9} \mathrm{Be}$ leading to the ${ }^{8} \mathrm{Be}$ g.s., shown in Fig. 3 by the outlined circles, exhibit a steep exponential rise as $R_{\min }$ decreases. The ${ }^{8} \mathrm{Be}$ g.s. component assigned to inelastic projectile excitation (outlined diamonds) has a much shallower slope. A calculation of Coulomb excitation reproduces the slope. This included $E 1$ excitation to the $1.684 \mathrm{MeV} 1 / 2^{+}$state in ${ }^{9} \mathrm{Be}$, which decays to the ${ }^{8} \mathrm{Be}$ g.s. with essentially $100 \%$ probability, and excitation to the $2.429 \mathrm{MeV} 5 / 2^{-}$state, which has a $6 \%$ branch to the ${ }^{8} \mathrm{Be}$ g.s. Taking the $B E 1(\uparrow)$ value to be $0.053 e^{2} \mathrm{fm}^{2}[10,11]$, the calculation required multiplication by a factor of 2 (grey diamonds) to reproduce the absolute probabilities. This may be due to $E 1$ excitation to other states which decay to the g.s. of ${ }^{8} \mathrm{Be}(E 2$ excitation depends too strongly on $R_{\min }$ to explain the data, as shown by the dashed line).

The dependence of the prompt breakup probability on $R_{\text {min }}$ is shown by the filled circles. The scatter of these

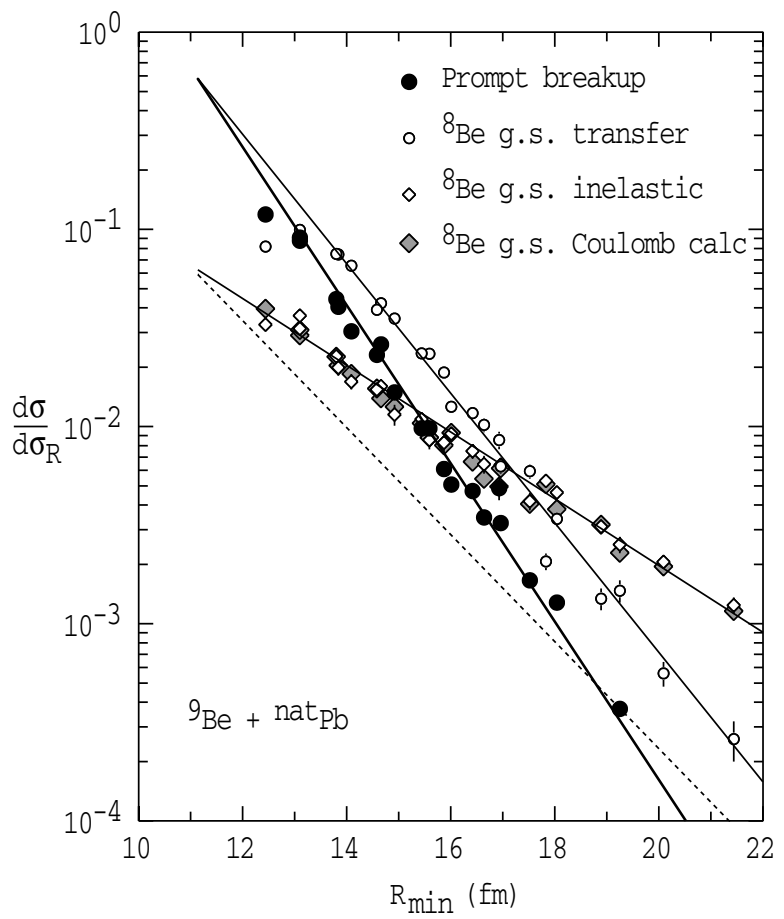

FIG. 3. Ratio of measured to Rutherford differential cross sections as a function of the distance of closest approach on a Coulomb trajectory $R_{\min }$, for each process. The full lines are exponential fits to the experimental data. The grey diamonds represent the calculated yield for Coulomb excitation to the first two excited states of ${ }^{9} \mathrm{Be}$, scaled up by a factor of 2 (see text). The dashed line shows the calculated slope for E2 Coulomb excitation. 
data from the fit is no more than for the ${ }^{8} \mathrm{Be}$ g.s. breakup. This indicates that although the $\alpha$ particles are more perturbed from the ${ }^{9} \mathrm{Be}$ trajectories by prompt breakup, typically by $\sim 10^{\circ}$, this is not sufficient to significantly scatter the deduced probabilities. The nature of the excitation process leading to prompt breakup can be investigated through the dependence of the probability on $R_{\min }$. The trend of calculations of E2 Coulomb excitation, normalized to the measured prompt breakup point at the largest $R_{\min }$, is indicated by the dashed line. The prompt breakup probabilities vary much more rapidly with $R_{\min }$ than this, and also more rapidly than the measured probabilities of neutron transfer to the ${ }^{8} \mathrm{Be}$ g.s. This suggests that prompt breakup is not associated with Coulomb excitation or neutron transfer, but appears to be predominantly associated with a nuclear process occurring close to the surface of the target nucleus.

Are the prompt breakup probabilities large enough to explain the observed suppression of complete fusion at above-barrier energies? This method of presenting the data allows a model-independent estimate of the prompt breakup probability at the fusion barrier radius. Extrapolating to the zero angular momentum $(l=0)$ fusion barrier radius (11.64 $\mathrm{fm} \mathrm{[3]),} \mathrm{the} \mathrm{probability} \mathrm{of} \mathrm{breakup}$ is 0.36 . The measured fusion suppression implies an incomplete fusion probability of $0.32 \pm 0.07$. The latter was, however, determined at beam energies above the average fusion barrier, where higher partial waves contribute. At $l=30$, for example (an angular momentum value which should have led to fusion at the higher energies in Fig. 2), the fusion barrier radius decreases by $0.5 \mathrm{fm}$, and the extrapolated prompt breakup probability increases to 0.58 . Breakup need not necessarily result in incomplete fusion, as both breakup fragments could be captured. It is thus necessary that the breakup probability be larger than the incomplete fusion probability, which seems to be the case. Although the subsequent motion and possible fusion of one or both of the breakup fragments needs to be modeled, the prompt breakup probability appears to be large enough to qualitatively explain the suppression of complete fusion found in the ${ }^{9} \mathrm{Be}+{ }^{208} \mathrm{~Pb}$ reaction. The strong dependence on the distance of closest approach suggests that prompt breakup results largely from a process close to the nuclear surface, and not the long-range Coulomb interaction.

What is the implication of this conclusion for the controversial topic [12] of fusion suppression in reactions of weakly bound nuclei with light target nuclei? In a simple picture, the breakup probability may be taken to be proportional to the gradient of the nuclear potential $\left(V_{N}^{\prime}\right)$ multiplied by a factor $f\left(r_{s}\right)$ which is dependent on the surface-to-surface separation $r_{s}$. The fit to the data gives $f\left(r_{s}\right) \propto \exp \left(-0.924 r_{s}\right)$. Assuming that this is not target specific, and that the probability of capture of both breakup fragments is the same for all target nuclei, the incomplete fusion probability of $0.32 \pm 0.07$ for $\mathrm{Pb}$ can be scaled to predict the incomplete fusion probability $P_{\text {ICF }}$ for any target:

$$
P_{\mathrm{ICF}}=0.32\left[V_{N^{\prime}} / V_{N^{\prime}}^{\mathrm{Pb}}\right] \exp \left(-0.924\left[r_{s}-r_{s}^{\mathrm{Pb}}\right]\right) .
$$

Here all quantities are evaluated at the appropriate fusion barrier radius. The Akyüz-Winther nuclear potential [13] was used to calculate both $V_{N}^{\prime}$ and $r_{s}$, resulting in $P_{\text {ICF }}$ scaling almost linearly with the charge of the target nucleus, at the same fraction of the fusion barrier energy. For ${ }^{9} \mathrm{Be}+{ }^{64} \mathrm{Zn}, P_{\text {ICF }}$ is predicted to be $0.13 \pm 0.03$, and for ${ }^{9} \mathrm{Be}+{ }^{19} \mathrm{~F}$ only $0.037 \pm 0.008$. These are consistent with recent measurements $[12,14,15]$ showing no fusion suppression, within $\sim 10 \%$ uncertainties, at energies above the fusion barrier.

From sub-barrier measurements of breakup in ${ }^{9} \mathrm{Be}+$ $\mathrm{Pb}$ reactions, it has been shown that prompt breakup appears to be associated with nuclear surface interactions, and may occur with high enough probability to explain the large suppression of complete fusion observed at above-barrier energies. The suppression factor for ${ }^{9} \mathrm{Be}$ reactions is predicted to be almost proportional to the charge of the target nucleus, making it most significant in reactions with heavy nuclei.

The work of M. D. was supported by the Australian Research Council. K. H. thanks N. Itagaki for useful discussions and the ANU for its hospitality during his stay.

[1] K. E. Rehm et al., Phys. Rev. Lett. 81, 3341 (1998), and references therein.

[2] J. J. Kolata et al., Phys. Rev. Lett. 81, 4580 (1998), and references therein.

[3] M. Dasgupta et al., Phys. Rev. Lett. 82, 1395 (1999).

[4] C. Signorini et al., Eur. Phys. J. A 5, 7 (1999).

[5] K. Hagino et al., Phys. Rev. C 61, 037602 (2000).

[6] R. J. Wooliscroft et al. (to be published).

[7] D. P. Stahel et al., Phys. Rev. C 16, 1456 (1977).

[8] R. J. Wooliscroft et al. (to be published).

[9] J. J. Kolata et al., Phys. Rev. C 65, 054616 (2002), and references therein.

[10] F. C. Barker, Can. J. Phys. 61, 1371 (1983).

[11] H. Utsunomiya et al., Phys. Rev. C 63, 018801 (2000).

[12] A. Mukherjee et al., Phys. Lett. B 526, 295 (2002), and references therein.

[13] Ö. Akyüz and A. Winther, in Nuclear Structure and Heavy-Ion Collisions, Proceedings of the International School of Physics "Enrico Fermi," Course LXXVII, edited by R. A. Broglia et al. (North-Holland, Oxford, 1981).

[14] S. B. Moraes et al., Phys. Rev. C 61, 064608 (2000).

[15] R. M. Anjos et al., Phys. Lett. B 534, 45 (2002). 